\title{
Localization in the Ground-State of the One Dimensional $X-Y$ Model with a Random Transverse Field
}

\author{
Abel Klein ${ }^{\star}$ and J. Fernando Perez ${ }^{\star \star \star \star \star \star \star}$ \\ Department of Mathematics, University of California, Irvine, Irvine, CA 92717, USA
}

\begin{abstract}
We consider the ground-state of the quantum spin model $H=$ $-J \Sigma_{\langle i,\rangle}\left[\sigma_{x}(i) \sigma_{x}(j)+\sigma_{y}(i) \sigma_{y}(j)\right]+\Sigma_{i} h_{i} \sigma_{z}(i)$ in one-dimension, where $\left\{h_{i}, i \in \mathbf{Z}\right\}$ are independent identically distributed random variables. By means of a Jordan-Wigner transformation the model is mapped into a free Fermi gas in the presence of a random external potential. We then use exponential localization of the one particle states to prove exponential decay for the spin-spin correlation functions.
\end{abstract}

\section{Introduction}

The Hamiltonian for the quantum $x-y$ model in the presence of a random transverse field is given by

$$
H=-J \Sigma_{\langle x, y\rangle}\left[\sigma_{1}(x) \sigma_{1}(y)+\sigma_{2}(x) \sigma_{2}(y)\right]+\Sigma_{x} h(x) \sigma_{3}(x),
$$

where $\sigma_{1}, \sigma_{2}, \sigma_{3}$, are the usual Pauli spin matrices, $x \in \mathbf{Z}^{d},\langle x, y\rangle$ denotes a pair of nearest neighbors in $\mathbf{Z}^{d}$, and the $h(x), x \in \mathbf{Z}^{d}$, are independent identically distributed random variables whose common probability distribution we will denote by $\mu$.

The quantum $x-y$ model in the presence of a random transverse field was shown by Ma, Halperin and Lee [1] to be relevant when studying the effect of disorder upon superfluidity. It was argued there that at high disorder localization should take place destroying the longrange order of the $x-y$ components of the spin system.

In this paper we consider the ground state of the one-dimensional model and show that, for any non-zero disorder, the elementary excitations of the system are localized and the correlation functions decay exponentially. This is to be compared

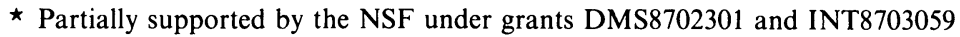

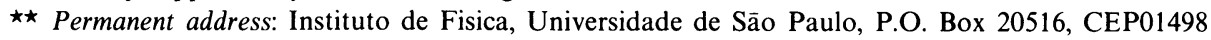
São Paulo, Brazil

$\star \star \star$ Partially supported by the CNPq under grant 303795-77FA
} 
with the polynomial decay obtained by Lieb, Schultz and Mattis [2] for zero transverse field.

As in [2] we "solve" the model by means of a Jordan-Wigner transformation [3] which maps the system into a free Fermi gas in the presence of a random external potential. In one dimensional the one particle states are localized $[4-7,10]$ for any non-zero disorder and this entails exponential decay for the one-particle Green's function with probability one.

Since the spin operators are non-local functions of the Fermi creation and annihilation operators, the study of the spin-spin correlations is much subtler than determining the ground state energy and the excitation spectrum $[2,8,9]$. However, using Wick's Theorem and convenient resummations we are able to show that the exponential fall-off of the one-particle Green's function yields exponential decay of the spin-spin correlation functions.

Given a positive integer $L$, we denote by $H_{L}$ the model Hamiltonian restricted to the box $\wedge_{L}=\mathbf{Z} \cap[-L, L]$, with free boundary conditions. The corresponding ground state, which we will show to be unique with probability one, will by denoted by $\psi_{L} \cdot\langle\cdot\rangle_{L}=\left(\psi_{L}, \cdot \psi_{L}\right)$ is the ground state expectation. We will also use $\sigma_{ \pm}=$ $\frac{1}{2}\left(\sigma_{1} \pm i \sigma_{2}\right)$.

Our result is

Theorem. Let $d=1$. Suppose the support of $\mu$ is not concentrated on a single-point and $\int|h|^{\eta} d \mu(h)<\infty$ for some $\eta>0$. Then for any $J$ there exists $m_{J}>0$ such that for almost every choice of the random transverse field $h$ we have

$$
\sup _{L}\left|\left\langle\sigma_{+}(x) \sigma_{-}(y)\right\rangle_{L}\right| \leqq C_{h} e^{-m_{J}|x-y|}
$$

for some $C_{h}<\infty$ and all $x, y \in \mathbf{Z}$.

Notice that we allow $\mu$ to have a delta function at zero, e.g., we can have $h(x)$ taking only the values 0 and 1 with nonzero probability.

This paper is organized as follows. In Sect. 2 we describe the model and review the Jordan-Wigner transformation. In Sect. 3 we discuss the properties of the one-particle Green's function and prove a folk theorem showing that exponential localization of states around the Fermi level implies exponential decay of correlation functions. In Sect. 4 we prove the theorem.

\section{The Model and its Ground State}

At each lattice site $x \in \mathbf{Z}$ we have a two dimensional space $\mathbf{C}^{2}=\mathscr{H}_{x}$ and the Pauli spin matrices

$$
\sigma_{+}(x)=\left(\begin{array}{ll}
0 & 1 \\
0 & 0
\end{array}\right), \quad \sigma_{-}(x)=\left(\begin{array}{ll}
0 & 0 \\
1 & 0
\end{array}\right), \quad \sigma_{3}(x)=\left(\begin{array}{rr}
1 & 0 \\
0 & -1
\end{array}\right) .
$$

For $L \in \mathbf{Z}, L>0$, we consider the finite system in $\wedge_{L}=\mathbf{Z} \cap[-L,+L]$. In the Hilbert space $\mathscr{H}_{L}=\bigotimes_{x \in \wedge_{L}} \mathscr{H}_{x}$, the Pauli space operators defined in the usual way satisfy the commutation rules

$$
\left[\sigma_{3}(x), \sigma_{ \pm}(y)\right]= \pm 2 \sigma_{ \pm}(x) \delta_{x y}
$$




$$
\left[\sigma_{+}(x), \sigma_{-}(y)\right]=\sigma_{3}(x) \delta_{x y} .
$$

We shall also make use of the operator

$$
n(x)=\frac{1+\sigma_{3}(x)}{2}=\sigma_{+}(x) \sigma_{-}(x) .
$$

The Hamiltonian in $\wedge_{L}$ with free boundary conditions is given (up to an energy shift) by:

$$
H_{L}=\Sigma_{x=-L}^{L} h(x) n(x)-J \Sigma_{x=-L}^{L-1}\left[\sigma_{+}(x) \sigma_{-}(x+1)+\sigma_{-}(x) \sigma_{+}(x+1)\right] .
$$

The external transverse fields $\{h(x), x \in \mathbf{Z}\}$ are independent identically distributed random variables with common distribution $d \mu(h)$, which we will always assume to satisfy the hypotheses of the Theorem.

Following [2] we introduce fermion creation and annihilation operators by the Jordan-Wigner [3] transformation. For $-L<x \leqq L$, let

$$
\begin{aligned}
a^{*}(x) & =\exp \left[i \pi \Sigma_{-L \leqq y<x} n(y)\right] \sigma_{+}(x), \\
a(x) & =\exp \left[i \pi \Sigma_{-L \leqq y<x} n(y)\right] \sigma_{-}(x)
\end{aligned}
$$

and,

$$
\begin{gathered}
a^{*}(-L)=\sigma_{+}(-L), \\
a(-L)=\sigma_{-}(-L) .
\end{gathered}
$$

The new operators satisfy canonical anticommutation relations (CAR):

$$
\begin{aligned}
\left\{a^{*}(x), a(y)\right\} & =\delta_{x y}, \\
\left\{a^{*}(x), a^{*}(y)\right\} & =\{a(x), a(y)\}=0
\end{aligned}
$$

$\forall x, y \in \wedge_{L}$. Here $\{A, B\}=A B+B A$.

The Hamiltonian $H_{L}$ can now be rewritten as:

$$
H_{L}=\Sigma_{-L \leqq x \leqq L} h(x) a^{*}(x) a(x)-J \Sigma_{x=-L}^{L-1}\left[a^{*}(x) a(x+1)+a^{*}(x+1) a(x)\right]
$$

which is the Hamiltonian for a gas of non-interacting spinless fermions in the presence of a random external potential $h(x)$.

We are thus led to consider the one-particle random Schrödinger operator

$$
H_{L}^{(1)}=-J \Delta_{L}+h
$$

in the Hilbert space $l^{2}\left(\wedge_{L}\right)$, where

$$
\left(\Delta_{L} \varphi\right)(x)=\Sigma_{y:|y-x|=1, y \in \wedge_{L}} \varphi(y)
$$

and $h$ is the multiplication operator

$$
(h \varphi)(x)=h(x) \varphi(x)
$$

with $\varphi \in l^{2}\left(\wedge_{L}\right)$. The operator $-\Delta_{L}$, apart from a trivial additive constant, is the usual lattice Laplacian with Dirichlet boundary conditions.

Let now $\varphi_{l}(x)$ and $\varepsilon_{l}, l=1, \ldots, 2 L+1$ denote the normalized eigenfunctions and respective eigenvalues of the Schrödinger operator. Notice that without loss of 
generality $\varphi_{l}(x)$ can be assumed to be real. We then introduce

$$
a_{l}^{*}=\Sigma_{x \in \wedge_{L}} \varphi_{l}(x) a^{*}(x), \quad a_{l}=\Sigma_{x \in \wedge_{L}} \varphi_{l}(x) a(x)
$$

for $l=1, \ldots, 2 L+1$, which also satisfy the CAR. The operator $H_{L}$ can now be written as:

$$
H_{L}=\Sigma_{l=1}^{2 L+1} \varepsilon_{l} a_{l}^{*} a_{l}
$$

so that its eigenvectors are

$$
\psi_{I}=\prod_{l \in I} a_{l}^{*} \Omega
$$

with eigenvalues

$$
E_{I}=\Sigma_{l \in I} \varepsilon_{l}
$$

where $I \subset\{1,2, \ldots, 2 L+1\}$ and $\Omega$ is the Fock (bare) vacuum:

$$
a(x) \Omega=0, \quad \forall x \in \wedge_{L} .
$$

In particular the ground state is given by

$$
\psi_{0} \equiv \psi_{I_{0}}=\prod_{l \in I_{0}} a_{l}^{*} \Omega
$$

where $I_{0}=\left\{l: \varepsilon_{l}<0\right\}$, with energy

$$
E_{0}=\Sigma_{l \in I_{0}} \varepsilon_{l} .
$$

More precisely, $\psi_{0, L}$ given by $(2.1)$ is the ground state of $H_{L}$ for all $L$ large enough, with probability one. For uniqueness of the ground state (and (2.1)) is equivalent to $\varepsilon_{l} \neq 0 l=1, \ldots, 2 L+1$. It follows from Theorem 2.1 in [10], by an application of the Borel Cantelli lemma, that, with probability one, zero is not in the spectrum of $H_{L}^{(1)}$ for all $L$ large enough.

More symmetrical expressions are obtained by introducing the usual "particlehole" operators;

$$
b_{l}=a_{l}^{*} \quad \text { if } \quad l \in I_{0}, \quad b_{l}=a_{l} \quad \text { if } \quad l \notin I_{0}
$$

which also satisfy CAR. For the new operators, the ground state is defined by the equations

$$
b_{l} \psi_{0}=0, \quad l=1, \ldots, 2 L+1
$$

\section{Exponential Decay of The Fermi Two-Point Function}

The two point function of the Fermi operators in the ground state can be computed to give

$$
\begin{aligned}
\left(\psi_{0}, a^{*}(x) a(y) \psi_{0}\right) & =\Sigma_{l, m} \varphi_{l}(x) \varphi_{m}(y)\left(\psi_{0}, a_{l}^{*} a_{m} \psi_{0}\right) \\
& =\Sigma_{l \in I_{0}} \varphi_{l}(x) \varphi_{l}(y)=P_{I_{0}}(x, y),
\end{aligned}
$$

where $P_{I_{0}}$ is the operator, in $l^{2}\left(\wedge_{L}\right)$, projecting into the subspace generated by $\left\{\varphi_{l}, l \in I_{0}\right\}$, and $P_{I_{0}}(x, y)$ its kernel. 
Let now $S$ denote the support of the single site probability distribution $d \mu(h)$. The spectrum $\sigma\left(H_{L}^{(1)}\right)$ of the operator $H_{L}^{(1)}$ is contained in the set

$$
\{S+2 J\} \cup\{S-2 J\}=\{E \in \mathbf{R}: \operatorname{dist}(E, S) \leqq 2 J\},
$$

since $-\Delta_{L}$ is a bounded operator with $\left\|-\Delta_{L}\right\|=2$.

Let us now assume that $|h(x)|>2 J+a$ for some $a>0$. In this situation the spectrum $\sigma\left(H_{L}^{(1)}\right)$ is contained in the set

$$
\left[-M_{L},-a\right] \cup\left[a, M_{L}\right]
$$

for some $M_{L}<\infty$, so that there is a gap of width $2 a$ around zero.

Under these assumptions the operator $P_{I_{0}}$ is given by the contour integral

$$
P_{I_{0}}=\frac{1}{2 \pi i} \oint_{c} R_{L}(z) d z,
$$

where

$$
R_{L}(z)=\left[\left(-J \Delta_{L}+h\right)-z\right]^{-1}
$$

and $C$ is a contour in the complex plane enclosing $\left[-M_{L},-a\right]$ while leaving $\left[a, M_{L}\right]$ on the complement of its interior.

Let $0<\delta_{0}<a / 2 J$ be chosen, we will take the contour (we write $z=u+i v$ ):

$$
\begin{aligned}
C & =\left\{0+i v ;-J\left(1+\delta_{0}\right) \leqq v \leqq 2 J\left(1+\delta_{0}\right)\right\} \\
& \cup\left\{u+i 2 J\left(1+\delta_{u}\right) ; u \leqq 0\right\} \cup\left\{u-i 2 J\left(1+\delta_{u}\right), u \leqq 0\right\},
\end{aligned}
$$

where $\delta_{u}=\delta_{0}\left(1+u^{2}\right)$. Notice that if $z+i v \in C$ we have $|z-h(x)| \geqq 2 J\left(1+\delta_{u}\right)$.

For $z \in C$, the expansion

$$
R_{L}(z)=(z-h)^{-1} \sum_{n=0}^{\infty}\left[\left(-J \Delta_{L}\right)(z-h)^{-1}\right]^{n}
$$

is convergent in the operator norm. In particular (3.2) implies that for the Green's function

$$
G_{L}(x, y ; z) \equiv\left\langle x\left|R_{L}(z)\right| y\right\rangle
$$

we have the absolute convergent expansion

$$
G_{L}(x, y ; z)=\Sigma_{w: x \rightarrow y} J^{|w|} \prod_{i=0}^{|w|} \frac{1}{z-h\left(w_{i}\right)}
$$

where the summation is taken over all walks $w$ on $\wedge_{L}$ going from $x$ to $y$, i.e. $w(n) \in \wedge_{L}$ for $0 \leqq n \leqq|w|, w(0)=x, w(|w|)=y$, where $|w|$ is any non-negative integer.

From (3.3), estimating the number of walks $w$ with a fixed length $|w|$ by $2^{|w|}$ we have

$$
\left|G_{L}(x, y ; z)\right| \leqq\left(\frac{1}{2 J \delta_{u}}\right)\left(\frac{1}{1+\delta_{u}}\right)^{|x-y|+1} .
$$

From (3.1) and (3.4) it then follows that

$$
\left|P_{I_{0}}(x, y)\right| \leqq \frac{1}{2 \pi} \oint_{C}\left|G_{L}(x, y ; z)\right||d z| \leqq \frac{l(C)}{2 J \delta_{0}}\left(\frac{1}{1+\delta_{0}}\right)^{|x-y|}
$$


for some constant $l(C)<\infty$. Therefore

$$
\left|P_{I_{0}}(x, y)\right| \leqq c_{0} e^{-m_{0}|x-y|}
$$

with constants $c_{0}=l(C) / 2 J \delta_{0}, m_{0}=\log \left(1+\delta_{0}\right)$ independent of $L$.

We are now going to drop the assumption of a gap. In this situation we have to make use of the localization results for one dimensional random Schrödinger operators.

If the probability distribution $\mu$ is absolutely continuous with a bounded density, it follows [4] that, with probability one, $P_{I_{0}}(x, y)$ is exponentially decaying for all $L$ large enough. For more general $\mu$ as in the Theorem, it follows from the results of $[10]$ by using $[5,6,7]$ that the exists $m>0$, such that with probability one, given $\varepsilon_{0}>0$,

$$
\sup _{|\varepsilon| \leqq \varepsilon_{0}}\left|G_{L}(x, y ; E+i \varepsilon)\right| \leqq c\left(h, \varepsilon_{0}\right) e^{-m|x-y|}
$$

for all $L$ large enough and all $x, y \in \mathbf{Z}$, with some constant $c\left(h, \varepsilon_{0}\right)<\infty$. We can then use (3.1) with the same contour $C$, where we take $\delta_{0}$ given by $m=\log \left(1+\delta_{0}\right)$ and choose $\varepsilon_{0}=2 J\left(1+\delta_{0}\right)$. As before, we obtain (3.5) with probability one for all $L$ large enough, with a different constant $c_{0}=c_{0}(h)$.

Thus, under the hypotheses of the Theorem, there exists $m_{J}>0$ such that, with probability one,

$$
\left|\left\langle a^{*}(x) a(y)\right\rangle_{L}\right| \leqq C_{h} e^{-m_{J}|x-y|}
$$

for all $L$ large enough and all $x, y \in \mathbf{Z}$, with some constant $C_{h}<\infty$.

\section{Correlation Functions}

In this section we discuss the asymptotic behavior of the correlation function $\left(\psi_{0}, \sigma_{+}(x) \sigma_{-}(y) \psi_{0}\right)$. We first write the non-local expressions for products of spin operators in terms of fermion operators: for $x<y$,

$$
\begin{aligned}
& \sigma_{+}(x) \sigma_{-}(y)=a^{*}(x) \prod_{x<z<y} \exp \{\operatorname{in}(z)\} a(y), \\
& \sigma_{-}(x) \sigma_{+}(y)=-a(x) \prod_{x<z<y} \exp \{\operatorname{in}(z)\} a^{*}(y) .
\end{aligned}
$$

If $\psi \in \mathscr{H}_{\wedge_{L}}$ is an eigenvector of the "total particle number" operator, i.e.

$$
\left(\Sigma_{x \in \wedge_{L}} n(x)\right) \psi=N \psi
$$

for some integer $N \geqq 0$, then

$$
\left(\psi, \sigma_{+}(x) \sigma_{-}(y) \psi\right)=e^{i \pi(N-1)}\left(\psi, \prod_{-L \leqq z<x} \exp \{i \pi n(z)\} a^{*}(x) a(y) \prod_{y<z^{\prime} \leqq L} \exp \left\{i \pi n\left(z^{\prime}\right)\right\} \psi\right)
$$

so that, for $x<y$

$$
\left(\psi, \sigma_{+}(x) \sigma_{-}(y) \psi\right)=-e^{i \pi(N-1)}\left(\psi_{x}^{-}, a^{*}(x) a(y) \psi_{y}^{+}\right)
$$

and

$$
\left(\psi, \sigma_{-}(x) \sigma_{+}(y) \psi\right)=e^{i \pi(N-1)}\left(\psi_{x}^{-}, a(x) a^{*}(y) \psi_{y}^{+}\right)
$$


where

$$
\psi_{x}^{-}=\prod_{-L \leqq z<x} \exp \{i \pi n(z)\} \psi
$$

and

$$
\psi_{y}^{+}=\prod_{y<z \leqq L} \exp \{i \pi n(z)\} \psi
$$

Next, following [2] we introduce the operators:

$$
\begin{aligned}
& A(z)=a^{*}(z)+a(z), \\
& B(x)=a^{*}(z)-a(z),
\end{aligned}
$$

which satisfy the anticommutation relations:

$$
\begin{aligned}
& \{A(x), A(y)\}=2 \delta_{x y}, \\
& \{B(x), B(y)\}=-2 \delta_{x y}, \\
& \{A(x), B(y)\}=0 .
\end{aligned}
$$

Moreover

$$
\exp [i \pi n(z)]=A(z) B(z) .
$$

Using (4.1), (4.2), (4.3) and (4.4) we get

$$
\begin{aligned}
\left(\psi, \sigma_{+}(x) \sigma_{-}(y) \psi\right) & =e^{i \pi(N-1)}\left(\psi_{x}^{-}, A(x) a(y) \psi_{y}^{+}\right) \\
& =e^{i \pi(N-1)}\left(\psi_{x}^{-}, A(x) B(y) \psi_{y}^{+}\right)+e^{i \pi(N-1)}\left(\psi_{x}^{-}, A(x) a^{*}(y) \psi_{y}^{+}\right) \\
& =-e^{i \pi(N-1)}\left(\psi_{x}^{-}, A(x) B(y) \psi_{y}^{+}\right)-\left(\psi, \sigma_{-}(x) \sigma_{+}(y) \psi\right),
\end{aligned}
$$

and so, for $x<y$

$$
\begin{aligned}
(\psi, & {\left.\left[\sigma_{+}(x) \sigma_{-}(y)+\sigma_{-}(x) \sigma_{+}(y)\right] \psi\right) } \\
= & e^{i \pi N}\left(\psi, \prod_{-L \leqq z<x}(A(z) B(z)) A(x) B(y) \prod_{y<z^{\prime} \leqq L}\left(A\left(z^{\prime}\right) B\left(z^{\prime}\right)\right) \psi\right)
\end{aligned}
$$

and

$$
\begin{aligned}
& \left(\psi,\left[\sigma_{+}(x) \sigma_{-}(y)-\sigma_{-}(x) \sigma_{+}(y)\right] \psi\right) \\
& \quad=e^{i \pi N}\left(\psi, \prod_{-L \leqq z<x}(A(z) B(z)) B(x) A(y) \prod_{y<z^{\prime} \leqq L}\left(A\left(z^{\prime}\right) B\left(z^{\prime}\right)\right) \psi\right) .
\end{aligned}
$$

A crucial fact now is that in both expressions (4.5) and (4.6) all operators involved anti-commute.

If we now take $\psi$ to be the ground state $\psi_{0}$ given by (2.1). We are in a position to apply Wick's Theorem. Indeed, if the operators $C_{1}, \ldots, C_{2 n}$ satisfy $\left\{C_{i}, C_{j}\right\}=0$, $i \neq j$, then

$$
\left(\psi_{0}, C_{1} C_{2} \cdots C_{2 n} \psi_{0}\right)=\Sigma_{P} \sigma(P)\left(\psi_{0}, C_{i_{1}} C_{j_{1}} \psi_{0}\right) \cdots\left(\psi_{0}, C_{i_{n}} C_{j_{n}} \psi_{0}\right),
$$

where the summation is done over all permutations $P=\left(i_{1}, j_{1}, i_{2}, j_{2}, \ldots, i_{n}, j_{n}\right)$ of $\{1,2, \ldots, 2 n\}, \sigma(P)$ being the corresponding signature. 
We then notice that

$$
\begin{aligned}
& \left(\psi_{0}, A(x) A(y) \psi_{0}\right)=\delta_{x y}, \\
& \left(\psi_{0}, B(x) B(y) \psi_{0}\right)=-\delta_{x y}, \\
& \left(\psi_{0}, B(x) A(y) \psi_{0}\right)=-\left(\psi_{0}, A(y) B(x) \psi_{0}\right) \equiv g_{L}(x, y),
\end{aligned}
$$

where

$$
\begin{aligned}
g_{L}(x, y) & =\left(\psi_{0}, a^{*}(x) a(y) \psi_{0}\right)+\left(\psi_{0}, a^{*}(y) a(x) \psi_{0}\right) \\
& =P_{I_{0}}(x, y)-P_{I_{0}}^{\perp}(x, y) .
\end{aligned}
$$

The operator $P_{I_{0}}-P_{I_{0}}^{\perp}$ is unitary in $l^{2}\left(\wedge_{L}\right)$, since $\left(P_{I_{0}}-P_{I_{0}}^{\perp}\right)^{2}=I$, and therefore the kernel $g_{L}(x, y)$ satisfies

$$
\Sigma_{z \in \wedge_{L}} g_{L}(x, z) g_{L}(z, y)=\delta_{x y} .
$$

Nơw $P_{I_{0}}^{\perp}(x, y)$ clearly satisfy the same bound (3.6) with possibly different constants, so that there are constants $c(h)$ and $m>0$ such that

$$
\left|g_{L}(x, y)\right| \leqq c(h) e^{-m|x-y|} .
$$

To simplify the notation let us rewrite (4.5) in the form

with

$$
\begin{aligned}
& \left(\psi_{0},\left[\sigma_{+}(x) \sigma_{-}(y)+\sigma_{-}(x) \sigma_{+}(y)\right] \psi_{0}\right) \\
& \quad=e^{i \pi N_{0}}\left(\psi_{0}, C_{2 l} C_{2 l-1} \cdots C_{1} C_{0} D_{0} D_{1} \cdots D_{2 k} \psi_{0}\right)
\end{aligned}
$$

$$
C_{0}=A(x), C_{1}=B(x-1), C_{2}=A(x-1), \ldots, C_{2 l}=A(-L)
$$

and

$$
D_{0}=B(y), D_{1}=A(y+1), D_{2}=B(y+1), \ldots, D_{2 k}=B(-L) .
$$

A similar expression holds for (4.6).

Since both the number of $C^{\prime} s$ and $D^{\prime} s$ are odd in every term contributing to (4.9) through Wick's theorem, there is a number $m \geqq 1$ of pairings of $C^{\prime} s$ with $D^{\prime} s$. We therefore write

$$
\begin{aligned}
\left(\psi_{0}, C_{2 l} \cdots C_{0} D_{0} \cdots D_{2 k} \psi_{0}\right) & =\sum_{m=1}^{\min \{2 k+1,2 l+1\}} \sum_{\substack{\left(i_{1}, j_{1}\right) \\
\vdots \\
\left({ }_{m}, j_{m}\right)}} \sigma_{i_{1}, \ldots, i_{m}} \sigma_{j_{1}, \ldots, j_{m}}\left\{\left(\psi_{0}, C_{i_{1}} D_{j_{1}} \psi_{0}\right) \cdots\left(\psi_{0}, C_{i_{k}} D_{j_{k}} \psi_{0}\right)\right. \\
& \left.\cdot\left(\psi_{0}, \prod_{i \neq i_{1}, \ldots, i_{m}} C_{i} \psi_{0}\right)\left(\psi_{0}, \prod_{j \neq j_{1}, \ldots, j_{m}} \psi_{0}\right)\right\},
\end{aligned}
$$

where $\sigma_{i_{1}, \ldots, i_{m}}$ and $\sigma_{j_{1}, \ldots, j_{m}}$ are such that

$$
\begin{aligned}
C_{2 l} C_{2 l-1} \cdots C_{1} C_{0} & =\sigma_{i_{1}, \ldots, i_{m}}\left(\prod_{i \neq i_{1} \cdots i_{m}} C_{i}\right) C_{i m} \cdots C_{i_{2}} C_{i_{1}}, \\
D_{0} D_{1} \cdots D_{2 k} & =\sigma_{j_{1}, \ldots, j_{m}} D_{j_{1}} D_{j_{2}} \cdots D_{j_{m}} \prod_{j \neq j_{1}, \ldots, j_{m}} D_{j} .
\end{aligned}
$$


Formula (4.10) is proved using Wick's Theorem and resumming all contractions not involving $i_{1}, \ldots, i_{m}$ and $j_{1}, \ldots, j_{m}$, again with Wick's Theorem.

To estimate (4.10) we first notice that

$$
\left|\left\langle\psi_{0}, \prod_{i \in I} C_{i} \psi_{0}\right\rangle\right| \leqq 1
$$

and

$$
\left|\left\langle\psi_{0}, \prod_{j \in J} D_{j} \psi_{0}\right\rangle\right| \leqq 1
$$

for any collection of indeces $I$ and $J$. This follows from the fact that both the right-hand side of (4.11) and (4.12) can be written as

$$
\pm\left(\psi_{0}, A\left(x_{1}\right) B\left(y_{1}\right) \cdots A\left(x_{n}\right) B\left(y_{n}\right) \psi_{0}\right)
$$

which by Wick's Theorem is given by the determinant

$$
\left(\psi_{0}, A\left(x_{1}\right) B\left(y_{1}\right) \cdots A\left(x_{n}\right) B\left(y_{n}\right) \psi_{0}\right)=\Sigma_{P} \sigma(P) \prod_{i=1}^{n} g\left(x_{i}, y_{P_{i}}\right)
$$

where the summation is taken over all permutations $(1, \ldots, n) \rightarrow(P 1, \ldots, P n)$.

Using Hadamard's Theorem

$$
|\operatorname{det} C|^{2} \leqq \prod_{i=1}^{n}\left(\sum_{j=1}^{n} C_{i j}^{2}\right)
$$

and (4.7) we get (4.11) and (4.12).

Therefore (4.10) can be estimated, by

$$
\begin{aligned}
& \left|\left(\psi_{0}, C_{2 l} \cdots C_{0} D_{0} \cdots D_{2 k} \psi_{0}\right)\right| \\
& \quad=\sum_{m=1}^{\min (2 k+1,2 l+1)} \sum_{\substack{0 \leqq i_{1}<i_{2}<\cdots<i_{k} \leqq 2 l \\
0 \leqq j_{1}<j_{2}<\cdots<j_{n} \leqq 2 k}}\left(\left(\psi_{0}, C_{i_{1}} D_{j_{1}} \psi_{0}\right) \cdots\left(\psi_{0}, C_{i_{m}} D_{j_{m}} \psi_{0}\right)\right) .
\end{aligned}
$$

This implies

$$
\begin{aligned}
& \left|\left(\psi_{0},\left[\sigma_{+}(x) \sigma_{-}(y)+\sigma_{-}(x) \sigma_{+}(y)\right] \psi_{0}\right)\right| \\
& \quad \leqq \sum_{m=1}^{\min \{x+L, L-y\}} \sum_{\substack{-L \leqq z_{1}<z_{2}<\cdots<z_{m} \leqq x \\
y \leqq z_{1}^{\prime}<z_{2}^{\prime}<\cdots z_{m}^{\prime} \leqq L}} \Sigma_{P}\left[\left|g_{L}\left(z_{1}, z_{P 1}^{\prime}\right)\right| \cdots\left|g_{L}\left(z_{m}, z_{P_{m}}^{\prime}\right)\right|,\right.
\end{aligned}
$$

the same estimate holding for $y<x$.

The number of pairs $\left(z_{i}, z_{j}^{\prime}\right)$ such that $\left|z_{i}-z_{j}^{\prime}\right|=|y-x|+R$ for some $R \geqq 0$ equals $R+1$. Using (4.8) we get the simple estimate

$$
\begin{aligned}
& \left|\left(\psi_{0},\left[\sigma_{+}(x) \sigma_{-}(y)+\sigma_{-}(x) \sigma_{+}(y)\right] \psi_{0}\right)\right| \\
& \quad \leqq \Sigma_{k=1}^{\infty}\left[\Sigma_{R=0}^{\infty} c(h) e^{-m|y-x|+R]}(R+1)\right]^{k} \\
& \quad=\Sigma_{k=1}^{\infty} e^{-m|y-x| k} c(h)^{k}\left(\Sigma_{R=0}^{\infty} e^{-m R}(R+1)\right)^{k} .
\end{aligned}
$$

For $|x-y|$ sufficiently large the right-hand side can be estimated yielding,

$$
\left|\left(\psi_{0},\left[\sigma_{+}(x) \sigma_{-}(y)+\sigma_{-}(x) \sigma_{+}(y)\right] \psi_{0}\right)\right| \leqq K(h) e^{-m|x-y|}
$$


with

$$
K(h)=d c(h) \frac{1}{1-d c(h) e^{-m|x-y|}},
$$

for a given constant $d<\infty$, thus concluding the proof.

Acknowledgements. A. K. is grateful to Daniel and Mathew Fisher for getting him interested in the problem and for useful discussions. A. K. also wants to express his debt to Claudio Albanese and Fabio Martinelli for many discussions on the multidimensional problem. Some of the insights gained in those discussions made their way into this paper.

\section{References}

1. Ma, M., Halperin, B. I., Lee, P. A.: Phys. Rev. B34, 3136-3143 (1986)

2. Lieb, E. H., Schultz, T. D., Mattis, D. C.: Ann. Phys. 16, 407-466 (1961)

3. Jordan, P., Wigner, E.: Z. Physik 37, 631 (1928)

4. Kunz, H., Souillard, B.: Commun. Math. Phys. 78, 201-246 (1980)

5. Fröhlich, J., Spencer, T.: Phys. 88, 151-184 (1983).

6. Fröhlich, J., Martinelli, F., Scoppola, E., Spencer, T.: Commun. Math. Phys. 101, 21-46 (1985)

7. von Dreifus, H., Klein, A.: A new proof of localization in the Anderson tight binding model, Commun. Math. Phys. 124, 285-299 (1989)

8. McCoy, B. M.: Phys. Rev. 173, 531-541 (1986)

9. Thompson, C. J.: One dimensional model short range forces. In: Phase transition and critical phenomena, Vol. I, Domb, C, Green, M. S. (eds.). London Academic Press 1967

10. Carmona, R., Klein, A., Martinelli, F.: Commun. Math. Phys. 108, 41-66 (1987)

Communicated by T. Spencer

Received May 12, 1989 